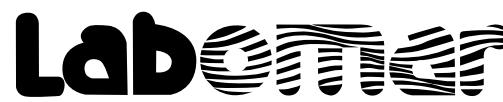

Arquivos de Ciências do Mar

\section{A PESCA DE MOLUSCOS EM AMBIENTES INTERMAREAIS NO OESTE DO ESTADO DO RIO GRANDE DO NORTE, BRASIL}

\author{
The mollusk harvesting in intertidal environments on western \\ Rio Grande do Norte State, Brazil
}

\author{
Ellano José da Silva ${ }^{1 *}$ e Inês Xavier Martins² \\ ${ }^{1}$ Instituto de Educação, Ciência e Tecnologia do Rio Grande do Norte, IFRN - Campus Macau. \\ Rua das Margaridas, 300 - Macau - RN \\ ${ }^{2}$ Laboratório de Moluscos - LABMOL, Centro de Ciências Biológicas e da Saúde - CCBS, Universidade Federal Rural do \\ Semi Árido - UFERSA. Av. Francisco Mota, 572. Presidente Costa e Silva. 59625-900. Campus Leste. \\ *Autor para contato. E-mail: ellanosilva7@gmail.com
}

\begin{abstract}
RESUMO
Os moluscos são um importante recurso pesqueiro extensivamente capturado por comunidades pesqueiras, porém estudos sobre sua explotação ainda são escassos no Nordeste brasileiro. O presente estudo teve como objetivo registrar as espécies de moluscos capturadas e comercializadas na costa Oeste do RN. Entre os anos de 2010 e 2013, foram amostradas 7 praias da região, onde ocorre a pescaria de moluscos. Pescadores de moluscos (marisqueiros) foram entrevistados e as espécies coletadas identificadas in situ. Foram identificadas nove espécies de moluscos, sendo os bivalves o grupo de maior representatividade. Algumas espécies são capturadas a penas para o consumo próprio, embora a maioria seja comercializada por valores de $\mathrm{R} \$ 4,00$ a 10,00 o quilograma. A captura das espécies ocorre ao longo do ano, com exceção do bivalve Mytella falcata que tem sua captura inviabilizada no período chuvoso. O polvo Octopus insularis e a ostra Crassostrea mangle (= Crassostrea rhizophorae) são os moluscos de maior valor econômico.
\end{abstract}

Palavras-chave: Mariscos, extrativismo, malacofauna, mariscagem, Bivalvia, Nordeste.

\section{ABSTRACT}

Molluscs are an important group extensively captured by fishing communities, however studies on their exploitation are still scarce in the Brazilian Northeastern. The present study aimed to

Recebido em: 13/9/2017

Aprovado em: 2/7/2017

Publicado online em: 20/1/2018 
record the malacofauna caught and traded on the west coast of the RN. Between the years of 2010 and 2013, 7 beaches of the region were sampled, where molluscs are harvested by artisanal fishey. Mollusc catchers were interviewed and the species collected by them were identified in situ. Nine mollusc species were identified, the bivalves were the most representative group. Some species are caught only for fishemen/fisherwomen own consumption, although most of them are traded for $R \$ 4.00$ to 10.00 per kilogram. The fishery takes place throughout the year, except for the bivalve Mytella falcata that has its capture unviable during the rainy season. The octopus Octopus insularis and the oyster Crassostrea mangle (= Crassostrea rhizophorae) have the highest prices.

Key words: Clams, extractive activities, malacofauna, shellfishery, Bivalvia, Northeastern Brazil.

\section{INTRODUÇÃO}

Pescarias de pequena escala constituem um importante componente socioeconômico na América Latina. Nessa região existem mais de 2.000 comunidades de pescadores artesanais e mais de 1 milhão de pessoas diretamente associadas a essa atividade (Bermudez \& Agüero, 1994). A pesca artesanal de pequena escala pode ser dividida em dois tipos: a destinada à extração de recursos pelágicos e a destinada à extração de espécies bentônicas ou demersais (Orensanz \& Jamieson, 1998).

A pesca artesanal de organismos bentônicos representa uma expressiva fonte de renda e subsistência para as comunidades tradicionais em zonas costeiras (Castilla \& Defeo, 2001). No entanto, muitos invertebrados são vulneráveis à pressão de pesca, particularmente, onde a tecnologia de captura não é desenvolvida para permitir a exploração de uma gama maior de outras espécies (Dalzell \& Adams, 1996).

Os moluscos constituem o $3^{\circ}$ grupo mais capturado pela pesca marinha, com produção mundial no ano de 2010 atingindo 20.000.000 de toneladas (FAO, 2010). Desse total, o Brasil foi responsável por quase 14.000 toneladas (IBAMA, 2012). Esses animais têm sido usados como recurso alimentar pelas populações humanas desde o Neolítico. E esta atividade tem acompanhado a própria ocupação da espécie humana no litoral brasileiro, o pode ser comprovado pela presença de sítios arqueológicos, conhecidos como sambaquis (Figuti, 1993). O interesse por esse grupo não se limita somente ao consumo humano. As conchas desses animais são amplamente utilizadas na fabricação de peças de artesanato e joias, ressaltando ainda mais seu valor econômico (Farias \& Rocha-Barreira, 2007).

No litoral brasileiro, diversas espécies de moluscos são capturadas de forma bastante rudimentar pelas comunidades costeiras, sem fazer uso de medidas de manejo que possam garantir um uso sustentável desses recursos (Araújo, 2001). Apesar da importância desse grupo para as comunidades que vivem da exploração dos recursos marinhos (Rönback, 1999; Dias et al., 2007), estudos sobre a captura de moluscos ainda são escassos, principalmente na Região Nordeste onde o conhecimento quanto à composição da malacofauna ainda é incipiente (Simone, 2008; Martinez, 2008).

Levando em consideração que a pesca artesanal é responsável por cerca de $50 \%$ de toda a produção extrativista do Brasil (IBAMA, 2008), conhecer as espécies alvo dessa modalidade de pesca é um fator essencial para criação de futuras medidas de manejo e compreensão desta atividade. O presente trabalho lista as espécies de moluscos alvo da 
pesca artesanal na faixa de intermarés, no Litoral Oeste potiguar, caracterizando sua captura e comercialização.

\section{MATERIAL E MÉTODOS}

A área de estudo contemplou a mesorregião Oeste do Rio Grande do Norte. Essa área é formada pela união de 62 municípios, sendo 4 litorâneos: Tibau, Grossos, Areia Branca e Porto do Mangue (Figura 1). Estes municípios possuem clima semiárido com período chuvoso de fevereiro a maio (IDEMA, 2008) e a produção pesqueira deste litoral abastece os mercados de Mossoró, a segunda maior cidade do estado (Observação pessoal).

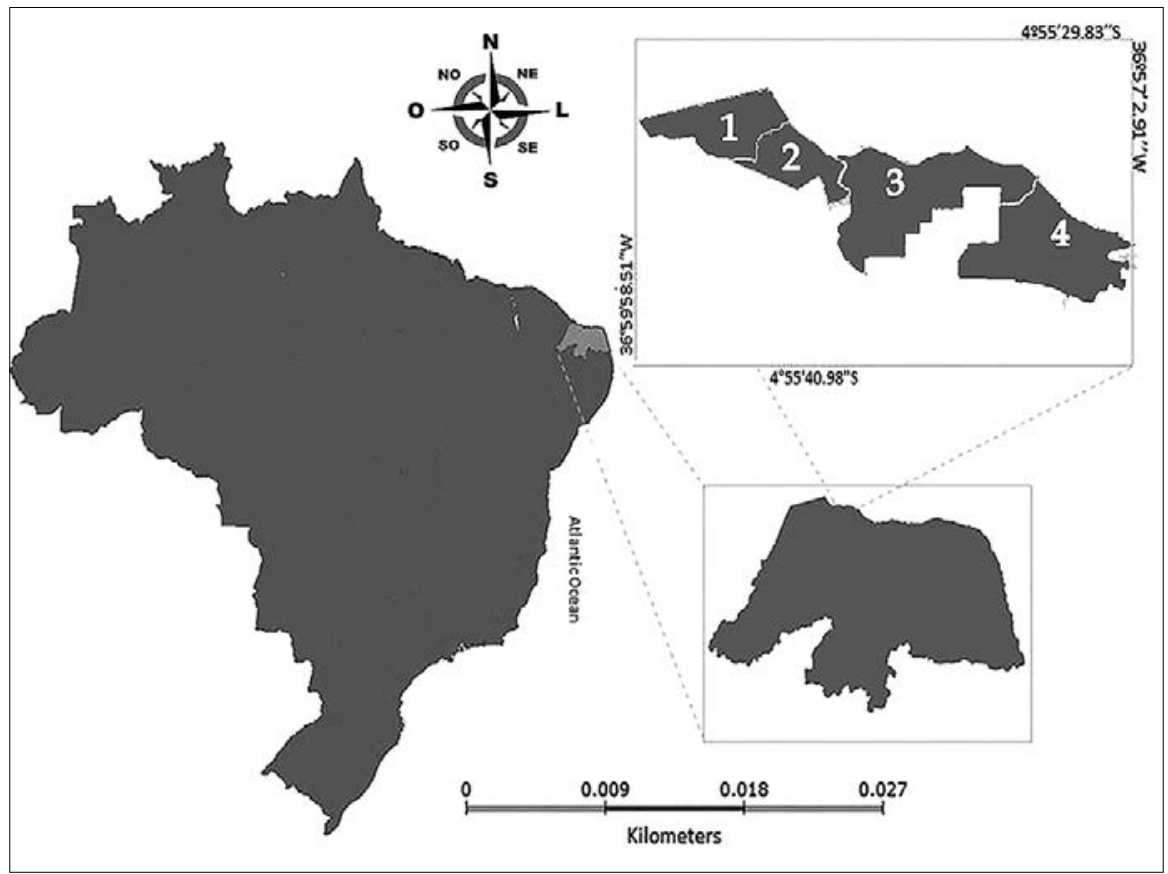

Figura 1 - Mapa da área de estudo com destaque ao Estado do Rio Grande do Norte e aos municípios amostrados. 1 - Tibau, 2 - Grossos, 3 - Areia Branca e 4 - Porto do Mangue. Modificado de: SILVA-JÚNIOR et al., 2013.

Foram visitadas as 7 principais praias, destes municípios, onde ocorre a captura de moluscos: 1 - Tibau: praia arenosa com algumas formações consolidadas provenientes de uma plataforma de abrasão; 2 - Pernambuquinho: sedimento arenoso com depósitos areno-lamosos; 3 - Barra: Desembocadura do rio Apodi-Mossoró, fundo composto de areia e argila; 4 - Baixa Grande: substrato arenoso com várias formações de arenito consolidado na faixa entremarés (Rebouças, et al., 2017); 5 - Ponta do Mel: praia arenosa com recifes de arenito e uma extensa plataforma de abrasão; 6 - São Cristóvão: predomínio de sedimento arenoso e recifes de arenito e 7 - Porto do Mangue: praia arenosa com grande influência do estuário do rio das Conchas.

Durante as expedições de coleta, realizadas de junho de 2010 a novembro de 2013, foi possível identificar in loco pescadores de molusco exercendo sua atividade (Figura 2), conhecidos localmente por "marisqueiros". Entrevistas com perguntas abertas de caráter investigativo foram realizadas com os marisqueiros com intuito de saber quais moluscos 
eram capturados, a época de captura, o destino das vendas e o preço de comercialização. Também foi possível identificar as espécies capturadas.

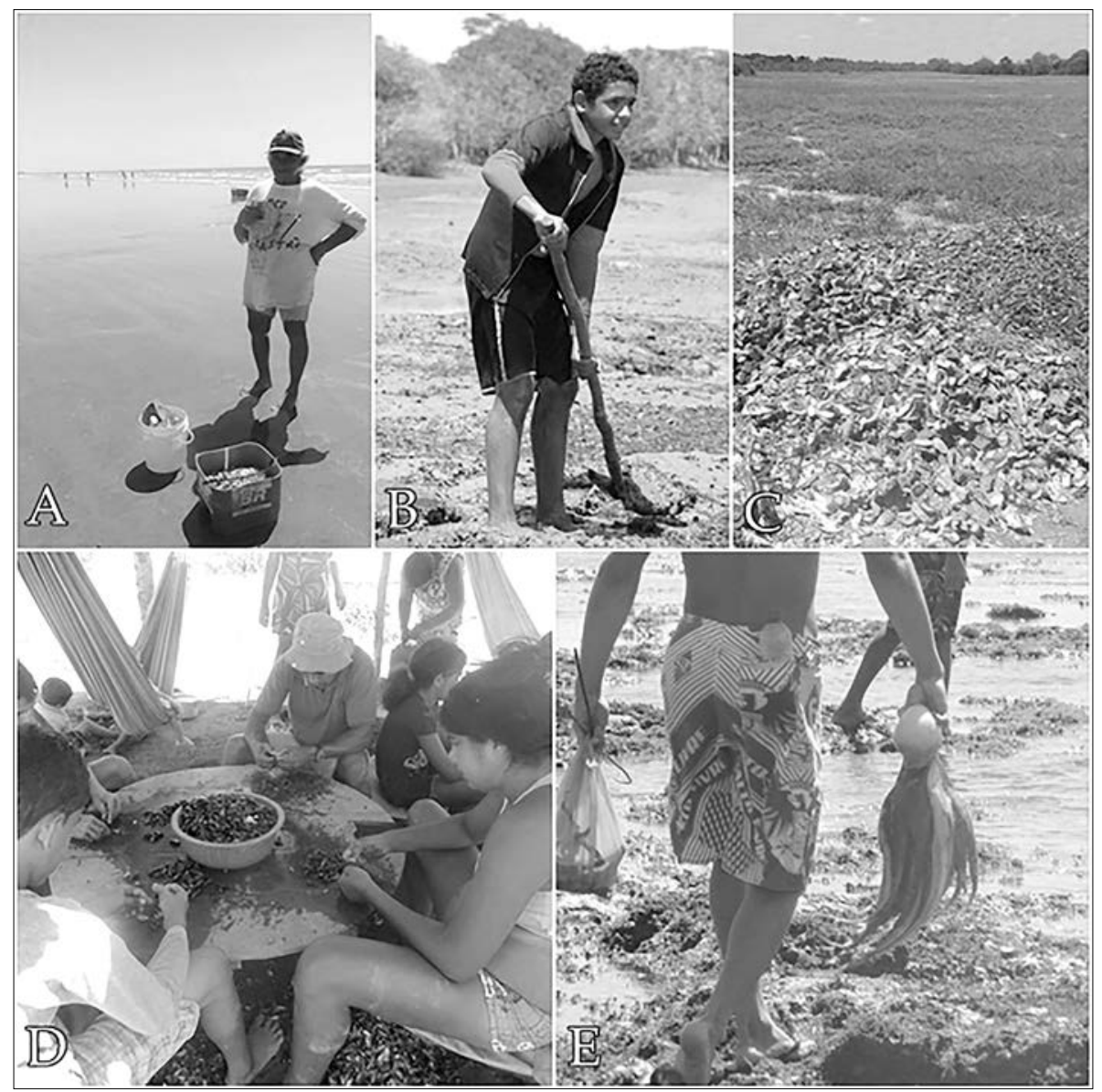

Figura 2 - Captura de Moluscos no oeste potiguar. A - Marisqueira na praia de Tibau; B - Criança removendo substrato lamoso para captura de M. falcata em Porto do Mangue; C - Descarte de conchas de ostras e sururus em P.M; D - Família realizando o beneficiamento do sururu ao ar livre em P.M.; E - A pesca de polvo em Areia Branca.

\section{RESULTADOS E DISCUSSÃO}

Foram entrevistadas 42 pessoas ao longo do período amostral. Estas eram selecionadas por estarem realizando a atividade de mariscagem no momento da amostragem.

Apesar de serem conhecidas mais de 60 espécies de moluscos na região (Silva et al., 2017), apenas nove espécies de moluscos são alvo da pesca, das quais os bivalves foram os mais representativos em número de espécies $(n=6)$, seguidos pelos gastrópodes $(n=2)$ e uma espécie de cefalópode $(n=1)$ (Tabela 1$)$.

A pesca de moluscos se restringiu às espécies que habitam águas rasas em regiões entremarés, que são facilmente acessadas pelos pescadores durante as marés baixas, principalmente as de sizígia, que expõe os ambientes onde estes organismos habitam facilitando sua coleta. 
Tabela 1 - Espécies de molusco capturadas por município, entre 2010 e 2013.

\begin{tabular}{lcccc}
\hline Espécie & Tibau & Grossos & Areia Branca & Porto do Mangue \\
\hline Donax striatus & $\mathrm{X}$ & $\mathrm{X}$ & $\mathrm{X}$ & \\
Mytella falcata & & $\mathrm{X}$ & & $\mathrm{X}$ \\
Mytella guyanensis & & & $\mathrm{X}$ \\
Crassostrea mangle & & $\mathrm{X}$ & $\mathrm{X}$ & $\mathrm{X}$ \\
Anomalocardia brasiliana & & $\mathrm{X}$ & $\mathrm{X}$ & \\
Tivela mactroides & & $\mathrm{X}$ & \\
Stramonita brasiliensis & & $\mathrm{X}$ & \\
Turbinela laevigata & & & & \\
Octopus insularis & & & & \\
\hline
\end{tabular}

\section{Bivalves}

Muitos bivalves habitam regiões de fácil acesso ao homem, possuem vida séssil ou sedentária, facilitando ainda mais sua captura. No ano de 2010, foram capturadas quase 9.000 toneladas de bivalves marinhos no Brasil (MPA, 2012). No Oeste Potiguar, as seis espécies de bivalves registradas (Tabela 1) pertencem a quatro famílias.

As ostras foram os bivalves que apresentaram maior valor comercial na área de estudo. No presente levantamento foi registrada uma única espécie de ostra: a Crassostrea mangle, embora nesta região também ocorre a espécie Crassostrea brasiliana que habita regiões de difícil acesso no estuário (Amaral \& Simone, 2014). As ostras são removidas das raízes-escoras do mangue vermelho (Rhizophorae mangle) com auxílio de facas. Apesar do pequeno tamanho atingido por esses animais em ambiente natural, as ostras são os bivalves com maior valor comercial.

Donax striatus, Anomalocardia brasiliana e Tivela mactroides foram os bivalves com maior ocorrência. Estes moluscos são comumente capturados pela população local para o consumo próprio, sendo a sua captura predominantemente realizada por mulheres. Esses bivalves formam densas populações, o que os torna susceptível a pesca (Ocaña, 2015; SilvaCavalcanti \& Costa, 2011; Turra et al., 2014).

O bivalve $A$. brasiliana é comercializado em diferentes escalas ao longo da costa brasileira, principalmente pelas comunidades litorâneas que a utilizam também na alimentação familiar (Boehs et al., 2008). No Oeste do Rio Grande do Norte, esta espécie é capturada na região estuarina de Areia Branca, Porto do Mangue e Grossos, neste último município encontra-se o estoque mais bem documentado (Rodrigues et al., 2013) e a única associação para o beneficiamento (cozimento para remoção da "carne" dos bivalves) de $A$. brasiliana da região (Ver Silva et al., 2014). A coleta manual é a principal forma de captura, a qual pode ser auxiliada por pás e os animais selecionados com monoblocos ou peneiras, variando de acordo com a comunidade pesqueira.

As espécies de sururu $M$. falcata e M. guyanensis são as únicas espécies de mytilídeos estuarinos que possuem importância econômica no Brasil (Pereira et al., 2003). Estes constituem no segundo táxon de bivalves mais capturado pela pesca. Na área de estudo, o principal mytilídeo capturado é $M$. falcata por esta formar densas agregações, com mais de mil indivíduos por $\mathrm{m}^{2}$, nas camadas superficiais do substrato. Estas densidades são muito superiores a $M$. guyanensis, que é capturada de forma esporádica pela população e formam pe- 
quenos agregados de dois a sete indivíduos semienterrados no substrato lamoso nos mangues, atingindo densidades de 16,6 a 264,4 indv./ $\mathrm{m}^{2}$ (Nishida \& Leonel, 1995; Pereira et al., 2003 ). Na região de Porto do Mangue ocorre uma forte pressão de pesca sobre a $M$. falcata, onde a depleção de alguns bancos chegou a ocorrer durante a realização do trabalho.

Famílias de pescadores de outras cidades costumam ir para esta localidade a fim de participar da mariscagem (Figura 2). Dentre os bivalves, apenas as espécies A. brasiliana e M. falcata possuem sua captura afetada pela sazonalidade (Tabela 2), não sendo coletadas durante os meses de maior precipitação pluviométrica (período chuvoso) que ocorre de janeiro a março. Os entrevistados relataram que os sururus tinham seu sabor alterado de forma negativa durante o período chuvoso.

Tabela 2 - Classificação taxonômica, período de captura e preço médio das espécies de molusco capturadas na área de estudo.

\begin{tabular}{|c|c|c|c|}
\hline Táxon & Espécie & Período de pesca & $\begin{array}{c}\text { Preço médio } \\
\text { (R\$) }\end{array}$ \\
\hline \multirow{6}{*}{ Bivalvia } & Donax striatus & Contínuo & $5,00 / \mathrm{kg}$ \\
\hline & Mytella falcata & Meses de seca* & $4,00 / \mathrm{kg}$ \\
\hline & Mytella guyanensis & - & - \\
\hline & Crassostrea mangle & Contínuo & $\begin{array}{c}4,00 \text { a } 8,00 \text { a } \\
\text { dúzia }\end{array}$ \\
\hline & Anomalocardia brasiliana & Meses de seca* & $10,00 / \mathrm{kg}$ \\
\hline & Tivela mactroides & Contínuo & $5,00 / \mathrm{kg}$ \\
\hline \multirow[t]{2}{*}{ Cephalopoda } & Octopus insularis & Contínuo & $10,00 / \mathrm{kg}$ \\
\hline & Turbinela laevigata & Contínuo & - \\
\hline Gastropoda & Stramonita brasiliensis & Contínuo & - \\
\hline
\end{tabular}

*Meses de seca: junho a janeiro

\section{Cefalópode}

Apenas uma espécie de cefalópode foi registrada, o polvo Octopus insularis. Esta espécie é considerada a de maior importância econômica no Nordeste brasileiro (Leite et al., 2009). O Rio Grande do Norte, atualmente, é um dos estados com as maiores capturas de polvo na região Nordeste, onde a pesca artesanal do O. insularis gera uma produção mé dia anual de 219 toneladas oriundas da frota artesanal (Vasconcelos, 2008), contudo as pescarias intermareais, como a relatada aqui, não entram nas estatísticas oficiais. A captura desta espécie só foi registrada em um dos municípios amostrados (Areia Branca), provavelmente pelo fato dessas praias possuírem recifes de arenito consolidado que favorecem a presença dos polvos.

A captura em ambientes intermareais é realizada de forma manual com auxílio de um gancho metálico, chamado de "bicheiro" e água sanitária que ao ser jogada na toca causa desconforto ao animal, fazendo-o sair de sua toca. Os polvos, quando comercializados, são vendidos frescos ou congelados, com o foco da produção voltado para o próprio município.

Dos moluscos capturados, o polvo é um dos que possui maior valor comercial, sua captura, segundo os entrevistados, ocorre o ano todo, sendo afetada apenas pelas marés. Ainda, de acordo com os entrevistados, maiores densidades de polvo ocorrem durante a lua cheia. 


\section{Gastrópodes}

Não existem dados acerca do extrativismo de moluscos gastrópodes no Brasil, mas mundialmente eles representam $2 \%$ dos moluscos capturados pela pesca (Leiva \& Castilla, 2002). Dentre as praias estudadas, somente em Ponta do Mel foram registradas capturas de espécies pertencentes ao táxon Gastropoda.

A espécie Stramonita brasiliensis é um gastrópode encontrado facilmente, uma vez que formam aglomerados em arrecifes na zona entre marés (Rios, 1994), possuem tamanho pequeno $(75 \mathrm{~mm})$ o que poderia torná-la não atraente para o consumo humano, porém este animal foi reportado pelos pescadores como um item muito apreciado em bares e restaurantes locais, entretanto não foi possível obter seu valor comercial. Este gastrópode é facilmente encontrado em formações rochosas formando agregações. Farias \& Rocha-Barreira (2008) também registraram o consumo de S. brasiliensis por uma comunidade costeira no Ceará, mas não reportam a comercialização deste molusco. A captura de $S$. brasiliensis parece ser esporádica, já que foi registrada apenas uma vez durante o período amostral.

O gastrópode Turbinella laevigata pode chegar a $20 \mathrm{~cm}$ de comprimento total (Rios, 1994) e é coletado facilmente durante a maré baixa, enterrados ou expostos no substrato arenoso. Foram encontrados pescadores coletando os animais manualmente e armazenando-os em baldes com capacidade de 5 litros. T. laevigata é capturada para consumo próprio, não sendo comercializada, na maioria das vezes, embora, de acordo com alguns pescadores, este gastrópode é um item alimentar procurado por alguns turistas. Alguns autores relatam que este molusco é utilizado na medicina tradicional para combater a impotência sexual e como afrodisíaco (Alves \& Dias, 2010; Neto, 2006), o que pode explicar em parte, a sua procura por parte de turistas. A pesca de gastrópodes é contínua, assim como a do polvo O. insularis, e em sua maior parte acontece apenas para o consumo dos próprios pescadores.

A pesca de moluscos na região estudada é basicamente artesanal, não sendo registrada na estatística oficial do IBAMA. O que pode levar a uma subestimação do montante de moluscos capturados no RN. Mesmo com um número de espécies consideravelmente baixo, os moluscos são um recurso pesqueiro importante para algumas comunidades de pescadores, seja por ser uma fonte de renda complementar durante o defeso de espécies de alto valor comercial como a lagosta, ou mesmo devido à facilidade de captura e acesso a esses organismos, possibilitando a atuação de mulheres e crianças na pesca.

Os resultados apresentados aqui constituem um passo primordial para o preenchimento da lacuna no estudo da pesca de moluscos, que representam o principal recurso pesqueiro coletado por muitas comunidades de pescadores (as).

Agradecimentos - A Universidade Federal Rural do Semi Árido (UFERSA) pelo auxílio financeiro e logístico na execução do trabalho;

- Aos dois revisores anônimos, que contribuíram com valiosas sugestões ao manuscrito;

- Ao professor Antônio Carlos B. S. Júnior, (Instituto Federal do Rio Grande do Norte) pelas sugestões dadas ao manuscrito.

\section{REFERÊNCIAS}

Alves, M.S.; Silva, M.A.; Júnior, M.M. Paranaguá, M.N. \& Pinto, S.L. Zooartesanato comercializado em Recife, Pernambuco, Brasil. Rev. Bras. Zooc. V. 8, p. 99-109, 2006. 
Alves, S.R.R.N. \& Dias, T.L.P. Usos de invertebrados na medicina popular no Brasil e suas implicações para conservação. Trop. Cons. Sci. v. 3, n. 2, p. 159-174, 2010.

Amaral, V.S. \& Simone, L.R. Revision of genus Crassostrea (Bivalvia: Ostreidae) of Brazil. J. of the Mar. Bio. Ass. of the UK, v. 94, n. 4, p. 811-836, 2014.

Araújo, C.M. Biologia reprodutiva do berbigão Anomalocardia brasiliana (Mollusca: Bivalvia, Veneridae) na Reserva Extrativista Marinha do Pirajubaé (REMAPI), Estado de Santa Catarina. Tese (Doutorado em Aquicultura) - Universidade de São Paulo, 2001. 203 f.

Bermudez A. \& Agüero M. Socioeconomic research on fisheries and aquaculture in Latin America. In: Charles, A.T., Brainerd, T.R., Bermudez, A., Montalvo, H.M. \& Pomeroy R.S. (eds.) Fisheries Socioeconomics in the Developing World. Regional Assessments and an Annotated Bibliography. Ottawa, ON, IDRC, p. 38-73. 1994

Boehs, G.; Absher, T.M. \& Cruz-Kaled, A.C. Ecologia populacional de Anomalocardia brasiliana (Gmelin, 1791) (Bivalvia, Veneridae) na Baía de Paranaguá, Paraná, Brasil. Bol. do Inst. de Pesca, São Paulo, v.34, n. 2, p. 259 - 270, 2008.

Castilla, J.C. \& Defeo, O. Latin American benthic shellfisheries: emphasis on co-management and experimental practices. Rev. in Fish Bio. and Fisher. v.11, p. 1-30, 2001

Dalzell, P. \& Adams, T.J.H. Sustainability and management of reef fisheries in the Pacific Islands. In 8th International Coral Reef Symposium, Panama City, 24-29. 1996

FAO (Food and Agriculture Organization of the United Nations) The state of world fisheries and aquaculture. p. 197. 2010.

Farias, M.F. \& Rocha-Barreira, C.A. Conchas de moluscos no artesanato cearense. Nave/ Labomar UFC, Fortaleza. 156p. 2007.

Figuti, L. O homem pré-histórico, o molusco e os sambaquis: considerações sobre a subsistência dos povos sambaquieiros. Rev. Mus. Arqueo. Etno./USP, São Paulo, v. 3, p. 67-80, 1993.

Instituto de Desenvolvimento Sustentável e Meio Ambiente do Rio Grande do Norte IDEMA RN. Perfil do seu município: Porto do Mangue. Natal - RN: vol. 10 p. 1-23. 2008

Instituto Brasileiro do Meio Ambiente e dos Recursos Naturais Renováveis - IBAMA. Estatística da pesca 2006 Brasil: grandes regiões e unidades da federação. Brasília, DF. 174p. 2008.

Leite, T.S.; Haimovici, M.; Mather, J. \& Lins Oliveira, J.E. Octopus insularis (Octopodidae) evidences of a specialized predator and a time-minimizing hunter. Mar. Biol., v. 156, n.11, p. 2355-2367, 2009.

Leiva, G.E. \& Castilla, J.C. A review of the marine gastropod fishery: evolution of catches, management and the Chilean experience. Rev. in Fish Bio. and Fisher, v. 11, p. 283 - 300, 2002.

Maia, I.C.C. \& Rocha-Barreira, C.A. Caracterização da atividade de captura de organismos da zona entre-marés, em recifes de arenito do litoral do Ceará, Brasil. Arq. Ciênc. Mar., v. 41, n.1, p. 67-73, 2008.

Martinez, A.S. Distribuição e abundância da malacofauna epibentônica no Parracho de Maracajaú, $R N$, Brasil. Dissertação de mestrado. (Mestrado em Bioecologia Aquática). Universidade Federal do Rio Grande do Norte, p. 43, Natal - RN. 2008.

Ministério da Pesca e Aquicultura (MPA). Boletim estatístico da pesca e aquicultura do Brasil (2010). MPA, Brasília, 129p. 2012 
Neto, E.M.C. Os moluscos na zooterapia: medicina tradicional e importância clínicofarmacológica. Biotemas, v.19, n. 3, p. 71-78, 2006.

Nishida, A.K. \& Leonel, R.M.V. Occurrence, population dynamics and habitat characterization of Mytella guyanensis (Lamarck, 1819) (Mollusca, Bivalvia) in the Paraiba do Norte River estuary. Bol. Inst. Oceanog. SP. v. 43, n.1, p. 41-49, 1995.

Ocaña, F.A. Growth and production of Donax striatus (Bivalvia: Donacidae) from Las Balsas beach, Gibara, Cuba. Rev. de Bio. Trop./Internat. J. of Trop. Bio. and Conserv., [S.1.], v. 63, n. 3, p. 639-646, 2015

Orensanz, J. \& Jamieson J. The assessment and management of spatially structured stocks. Can. Spec. Pub. of Fisher. and Aqua. Scienc. v. 125, n. 1, p. 441-459, 1998.

Pereira, O.M.; Hilberath, R.C.; Ansarah, P.R.A.C. \& Galvão, M.S.N. Estimativa da produção de Mytella falcata e de M. guyanensis em bancos naturais do estuário de Ilha Comprida - SP - Brasil. Bol. do Inst. de Pesca, v. 29, n. 2, p.139 -149, 2003.

Rebouças, L.O.S; Gomes, R.B; Da Silva, E.J. \& Martins, I.X. Variação espaço-temporal da malacofauna de uma praia do Rio Grande do Norte, Nordeste do Brasil. ActaFish, v.5, n. 2, p. 29-37, 2017

Rios, E. C. Seashells of Brazil. Rio Grande: Editora da Fundação Universidade do Rio Grande, $2^{a}$ ed. 492 p. 1994.

Rodrigues, A.M.L.; Borges-Azevedo, C.M.; Costa, R.S. \& Henry-Silva, G.G. Population structure of the bivalve Anomalocardia brasiliana, (Gmelin, 1791) in the semi-arid estuarine region of northeastern Brazil. Braz. Jour. of Biol. [online]. 2013, vol.73, n.4. 2013.

Rönback, P. The ecological basis for economic value of seafood production supported by mangrove ecosystems. Ecol. Econ., v. 29, p. 235-252, 1999.

Silva, G.H.G; Carolsfeld, J. \& Gálvez, A.O. Gente da Maré: Aspectos ecológicos e socieconômicos da mariscagem no nordeste brasileiro. EdUFERSA, p. 420, Mossoró - RN, 2014.

Silva, G.H.G; Martins, I.X; Rabelo, E.F. \& Silva, E.J. Guia Ilustrado de Moluscos do Litoral Oeste Potiguar. EdUFERSA, p. 177, Mossoró - RN, 2017.

Silva-Cavalcanti, J.S. \& Costa, M.F. Fisheries of Anomalocardia brasiliana in Tropical Estuaries. Pananjas, v. 6, n. 2, p. 86-99, 2011.

Simone, L.R.L. Status quo da Malacologia marinha no Brasil. Bol. Ass. Bras. Biol. Mar., v. 3, n. 1, p. 4-7. 2010.

Silva-Júnior, C.A.B.; Araújo, M.E. \& Feitosa, C.V. Sustainability of capture of fish bycatch in the prawn trawling in northeastern Brazil. Neotrop. Ichthyol., v. 11, n. 1, p. 133-142, 2013.

Turra, A.; Petracco, M.; Amaral, A.C.Z. \& Denadai, M.R. Population biology and secondary production of the harvested clam Tivela mactroides (Born, 1778) (Bivalvia, Veneridae) in Southeastern Brazil. Mar. Ecol., v. 36, n. 2, p. 221-234, 2014.

Vasconcelos, J.A.A pesca de polvo no Rio Grande do Norte. Polvo News 4, 3-3. 2008. 